\title{
Color Image Transmission in SPC Channel Encoded Multiuser 5G Compatible Pattern Division Multiple Access (PDMA) Wireless Communication System
}

\author{
Afrin Naher, Shaikh Enayet Ullah \\ Department of Applied Physics and Electronic Engineering, University of Rajshahi, Rajshahi, Bangladesh \\ Email address: \\ afrinnaher57@gmail.com (A. Naher), enayet_apee@ru.ac.bd (S. E. Ullah) \\ To cite this article: \\ Afrin Naher, Shaikh Enayet Ullah. Color Image Transmission in SPC Channel Encoded Multiuser 5G Compatible Pattern Division Multiple \\ Access (PDMA) Wireless Communication System. Advances in Wireless Communications and Networks. Vol. 4, No. 1, 2018 , pp. $17-29$. \\ doi: 10.11648/j.awcn.20180401.14
}

Received: July 26, 2018; Accepted: August 16, 2018; Published: September 13, 2018

\begin{abstract}
Pattern Division Multiple Access (PDMA) is a novel non-orthogonal multiple access scheme proposed to meet up the diverse demands on high capacity and large number of connections in the fifth generation $(5 \mathrm{G})$ wireless networks. PDMA uses the characteristic pattern to define the sparse mapping from data to a group of resources, and the sparsity of the pattern gives impacts on the capacity performance and detection complexity. The present thesis emphasizes greatly to develop a simulation model for the 5G compatible downlink Pattern Division Multiple Access (PDMA) scheme implemented wireless communication system. The simulated system incorporates a modern channel coding $((3,2)$ SPC) and different low order digital modulation (4-QAM, QPSK and DQPSK) schemes. With implementation of $\mathrm{G}^{[2,3]}$ PDMA pattern matrix for three users and considering total transmitted power of $40 \mathrm{Watt}, 50 \%, 30 \%$ and $20 \%$ of total transmitted power have been assigned to first, second and third users and additionally phase shifting factors have been applied in power scaled transmitted signals of second and third users to resolve ambiguity in individual data separation. On consideration of both synthetically generated binary data stream and color image transmission in AWGN and Rayleigh fading channel, it is observable from MATLAB based simulation study that the $((3,2)$ SPC) channel encoded simulated system is very much robust and effective in retrieving transmitted data. The impact of applicability of 2-D median image filtering technique in processing of three different identically sized color images and enhancement of image quality has clearly been observed. This thesis elucidates the benefits of multi-user data transmission in power domain.
\end{abstract}

Keywords: PDMA, $((3,2)$ SPC $), G^{[2,3]}$ PDMA Pattern Matrix, AWGN and Rayleigh Fading Channel, 2-D Median Image Filtering Technique

\section{Introduction}

Multiple access scheme is generally regarded as the landmark of each generation of mobile communication systems. In the first generation (1G), Frequency division multiple access (FDMA) was used. Time division multiple access (TDMA) and code division multiple access (CDMA) were introduced in the second generation $(2 \mathrm{G})$ and the third generation $(3 \mathrm{G})$ respectively. Orthogonal frequency division multiple access (OFDMA) is considered as the key component of the fourth generation $(4 \mathrm{G})$. The multiple access schemes separate users in orthogonal resources such as frequency, time, or code domain resources. These orthogonal multiple access (OMA) schemes make it possible to build the system with low complexity, however they are unable to achieve the capacity boundary of a multi-user channel. The fifth generation $(5 \mathrm{G})$ needs to support much higher capacity and also provide much larger number of connected users. These requirements are rather challenging and it is difficult to satisfy the requirements by using OMA schemes. Nonorthogonal multiple access (NOMA) has thus been considered as a promising candidate to meet the requirements in connection numbers and system capacity of 5G. Signal of multiple users are superposed and advanced detection algorithm are employed to separate the superposed signal. Theoretically, NOMA is optimal in terms of achieving the 
capacity boundary $[1,2]$. In the previous $3 \mathrm{G}$ WCDMA wireless communication system, NOMA has been used. In the uplink of WCDMA, data symbols are spread by long spreading codes and multiple users transmit their spread signals on the same frequency and time resources. Since long spreading codes are used, only linear detection algorithm is possible at the receiver due to the complexity of non-linear detection algorithm. As a result, non-orthogonal transmission of this type is demonstrated to be inefficient in terms of spectrum efficiency [3]. A number of NOMA techniques are studied in both industry and academy, such as interleave division multiple access (IDMA) [4], bit division multiplexing (BDM) [5], sparse code multiple access (SCMA) [6] and multi-user sharing access (MUSA) [7] etc. With IDMA, different users are distinguished by userspecific interleavers. BDM was first proposed to provide differentiated services for broadcasting system. It extends the multiplexing from symbol level to bit level. Both SCMA and MUSA are based on code domain superposition [8]. In 2016, Chen and et.al., suggested various nonlinear detection algorithms such as successive interference cancellation (SIC), maximum a posteriori (MAP) or maximum likelihood (ML) in receiving section for separating users being multiplexed on the same resource. Pattern Division Multiple Access (PDMA) is a code pattern based NOMA scheme. The PDMA patterns are designed to offer different order of transmission diversity, so that disparate diversity order between multiple users could be introduced to alleviate the error propagation problem of SIC receiver. In transmission diversity, multiple copies of a signal are transmitted from independent resources to avoid transmission error due to deep fading on one resource. The resources could be time, frequency or spatial resource. The PDMA pattern is also extended to include power scaling and phase shifting to harvest additional constellation shaping gain. In PDMA, nonorthogonal transmission is realized through multiplexing data of multiple users onto the same resource group with different PDMA pattern.

\section{PDMA Pattern Design and Signal Detection}

Users multiplexed PDMA patternson the same resource group can construct a PDMA pattern matrix. Each column of the PDMA pattern matrix represents a PDMA pattern. Properties of PDMA pattern matrix such as dimension and level of sparsity contribute both complexity and system performance. In our present study, a $2 \times 3$ PDMA pattern matrix has been used where 3 users are multiplexed on two resource elements (REs). The PDMA pattern in such case can be written as:

$$
G_{P D M A}^{[2,3]}=\left[\begin{array}{lll}
1 & 1 & 0 \\
1 & 0 & 1
\end{array}\right]
$$

Taking PDMA pattern $G_{P D M A}^{[2,3]}$, transmitted data of 3 users are mapped onto two REs. The transmission signal on these REs can be expressed as:

$$
\left[\begin{array}{l}
v_{1} \\
v_{2}
\end{array}\right]=\left[\begin{array}{lll}
1 & 1 & 0 \\
1 & 0 & 1
\end{array}\right]\left[\begin{array}{l}
x_{1} \\
x_{2} \\
x_{3}
\end{array}\right]
$$

where, $v_{j}$ is the transmission signal on the $j^{\text {th }} \mathrm{RE}$, and $\mathrm{x}_{\mathrm{k}}$ is the modulation symbol of the $\mathrm{k}^{\text {th }} \mathrm{user}$

Unlike orthogonal transmission, transmission signal on each RE is linear combination of multiple modulation symbols:

$$
\begin{aligned}
& \mathrm{V}_{1}=\mathrm{x}_{1}+\mathrm{x}_{2} \\
& \mathrm{~V}_{2}=\mathrm{x}_{1}+\mathrm{x}_{3}
\end{aligned}
$$

To resolve ambiguity in proper detection of user's own data, power scaling and phase shifting are introduced in PDMA pattern matrix. Specifically, before two users' symbols are mixed, a power scaling factor and a phase shifting factor are applied:

$$
v=\sqrt{\beta} x_{1} e^{j \phi}+\sqrt{1-\beta} x_{2}
$$

where, $\beta$ is the power scaling factor and $\phi$ is the phase shifting factor. In this present study, merely phase shifting factor has been applied to data of user \#2 and user \#3. As each user is equipped with a single antenna and base station is equipped with two antennas, the fading channel matrix is $2 \times 3$ sized and it is represented as:

$$
H_{C H}=\left[\begin{array}{lll}
h_{11} & h_{12} & h_{13} \\
h_{21} & h_{22} & h_{23}
\end{array}\right]
$$

The PDMA equivalent channel response matrix $\mathrm{H}$ can be written as:

$$
\begin{aligned}
\mathrm{H}=H_{C H} & \Theta G^{[2,3]} \text { PDMA } \\
& =\left[\begin{array}{lll}
h_{11} & h_{12} & h_{13} \\
h_{21} & h_{22} & h_{23}
\end{array}\right]\left[\begin{array}{lll}
1 & 1 & 0 \\
1 & 0 & 1
\end{array}\right]=\left[\begin{array}{lll}
h_{11} & h_{12} & 0 \\
h_{21} & 0 & h_{23}
\end{array}\right]
\end{aligned}
$$

where, $\Theta$ has been considered to represent element-wise product of two matrices. In PDMA equivalent channel response matrix $\mathrm{H}$, the channel coefficients of first, second and third columns are applicable to user \#1, user \#2 and user\#3 [9]. As signal transmitted from Base station from two antennas use different resource (frequency), the signal received at user \#1 from second antenna of Base station is neglected. The signal received at the user \#1, user \#2 and user \# 3 can be written as:

$$
\begin{aligned}
& \mathrm{y} 1=\mathrm{h}_{11}\left(\mathrm{x}_{1}+\mathrm{x}_{2}\right) \\
& \mathrm{y} 2=\mathrm{h}_{12}\left(\mathrm{x}_{1}+\mathrm{x}_{2}\right) \\
& \mathrm{y} 3=\mathrm{h}_{23}\left(\mathrm{x}_{1}+\mathrm{x}_{3}\right)
\end{aligned}
$$


From equation (7) through equation (9), it is observable that in every cases, another user's data are treating as interference which are needed to be eliminated. Primarily, digitally modulated complex symbol vectors for three users $\hat{x}_{1}, \hat{x}_{2}$ and $\hat{x}_{3}$ are scaled in power such that the average power for each user is made to unity. If $\mathrm{P}$ be the total transmitted power and $\beta_{1}, \beta_{2}$ and $\beta_{3}$ are the power scaling factors $(0.5,0.3$ and 0.2) of three users, $\phi_{2}$ and $\phi_{3}$ are phase scaling factors for user \#2 and user \#3, the power and phase scaled signal vector of the three users can be written as:

$$
\begin{aligned}
& \hat{\hat{x}}_{1}=\sqrt{P \beta_{1}} \hat{x}_{1} \\
& \hat{\hat{x}}_{2}=\sqrt{P \beta_{2}} \hat{x}_{2} e^{j \varphi_{2}} \\
& \hat{\hat{x}}_{3}=\sqrt{P \beta_{3}} \hat{x}_{3} e^{j \varphi_{3}}
\end{aligned}
$$

On applying OFDM modulation to each data vector presented in Equation (10), the transmitted signal vectors for three users $x_{1}, x_{2}$ and $x_{3}$ are produced. In receiving section of each user, the AWGN noise contaminated Rayleigh faded signal is undergone OFDM demodulation. The OFDM demodulated signal vectors $\mathrm{x}_{10}, \mathrm{x}_{20}$ and $\mathrm{x}_{30}$ are channel equalized and can be written as:

$$
\begin{gathered}
\mathrm{x}_{11}=\left(\mathrm{h}_{11}\right)^{-1} \mathrm{x}_{10} \\
=\sqrt{P \beta_{1}} \hat{x}_{1}+\sqrt{P \beta_{2}} \hat{x}_{2} e^{j \varphi_{2}} \\
\mathrm{x}_{22}=\left(\mathrm{h}_{12}\right)^{-1} \mathrm{x}_{20} \\
=\sqrt{P \beta_{1}} \hat{x}_{1}+\sqrt{P \beta_{2}} \hat{x}_{2} e^{j \phi_{2}} \\
\mathrm{x}_{33}=\left(\mathrm{h}_{23}\right)^{-1} \mathrm{x}_{30} \\
=\sqrt{P \beta_{1}} \hat{x}_{1}+\sqrt{P \beta_{3}} \hat{x}_{3} e^{j \phi_{3}}
\end{gathered}
$$

In equation (11) through (13), $\hat{x}_{1}, \hat{x}_{2}$ and $\hat{x}_{3}$ are the detected power scaled complex modulated symbol vectors for three users. These signal vectors are assumed to have average power of unity which are further rescaled to retrieve original signal vectors. In equations (11), (12) and (13), $\sqrt{P \beta_{2}} \hat{x}_{2} e^{j \phi_{2}}, \sqrt{P \beta_{1}} \hat{x}_{1}$ and $\sqrt{P \beta_{1}} \hat{x}_{1}$ are the interference terms, denoting $\mathrm{X}=\left\{\mathrm{x}^{(1)}, \mathrm{x}^{(2)}, \mathrm{x}^{(3)}\right.$ and $\left.\mathrm{x}^{(4)}\right\}$ is the power scaled (unity average power) signal alphabet of 4QAM/QPSK/DQPSK and using ML decoding, the detected interfering complex symbols $\bar{x}_{1}, \bar{x}_{2}$ and $\vec{x}_{3}$ contained in interference term for user \#1, user \#2 and user \#3 can be obtained from:

$$
\begin{gathered}
\bar{x}_{1}=\arg \min \left|\mathbf{x}_{11}-\sqrt{\mathbf{P} \beta_{1}} \hat{\mathbf{x}}_{\mathbf{1}}-\sqrt{\mathbf{P} \boldsymbol{\beta}_{2}} \mathbf{x}^{(\mathbf{k})} \mathbf{e}^{\mathbf{j} \varphi_{2}}\right|, \mathbf{k}=\mathbf{1 , 2 , 3 , 4} \\
\mathbf{x}^{(\mathbf{k})} \in \mathbf{X}
\end{gathered}
$$

$$
\begin{gathered}
\bar{x}_{2}=\arg \min \left|\mathbf{x}_{22}-\sqrt{\mathbf{P} \beta_{1}} \mathbf{x}^{(\mathbf{k})}-\sqrt{\mathbf{P} \beta_{2}} \hat{\mathbf{x}}_{22} \mathbf{e}^{\mathbf{j} \varphi_{2}}\right|, \mathbf{k}=\mathbf{1 , 2 , 3 , 4} \\
\mathbf{x}^{(\mathbf{k})} \in \mathbf{X} \\
\overline{\mathbf{x}}_{3}=\arg \min \left|\mathbf{x}_{33}-\sqrt{\mathbf{P} \beta_{1}} \mathbf{x}^{(\mathbf{k})}-\sqrt{\mathbf{P} \beta_{3}} \hat{\mathbf{x}}_{33} \mathrm{e}^{\mathbf{j} \varphi_{3}}\right|, \mathbf{k}=\mathbf{1 , 2 , 3 , 4} \\
\mathbf{x}^{(\mathbf{k})} \in \mathbf{X}
\end{gathered}
$$

The detected power scaled complex symbols $\hat{\hat{x}}_{1}, \hat{\hat{x}}_{2}$ and $\hat{\hat{x}}_{3}$ for three users can be written as:

$$
\begin{aligned}
& \hat{\hat{x}}_{1}=\left(\mathrm{x}_{11}-\sqrt{P \beta_{2}} \bar{x}_{1} e^{j \varphi_{2}}\right) /\left(\sqrt{P \beta_{1}}\right) \\
& \hat{\hat{x}}_{2}=\left(\mathrm{x}_{22}-\sqrt{P \beta_{1}} \bar{x}_{2}\right) /\left(\sqrt{P \beta_{2}} e^{j \varphi_{2}}\right) \\
& \hat{\hat{x}}_{3}=\left(\mathrm{x}_{33^{-}} \sqrt{P \beta_{1}} \bar{x}_{3}\right) /\left(\sqrt{P \beta_{3}} e^{j \varphi_{3}}\right)
\end{aligned}
$$

On multiplying Equation (17) through Equation (19) with normalization factor $\mathrm{NF}(=\sqrt{2})$, the original complex digitally modulated data symbols for user \#1, user \#2 and user\#3 are achieved viz.

$$
\begin{aligned}
& \hat{\hat{\hat{x}}}_{1}=N F \hat{\hat{x}}_{1} \\
& \hat{\hat{\hat{x}}}_{2}=N F \hat{\hat{x}}_{2} \\
& \hat{\hat{\hat{x}}}_{3}=N F
\end{aligned}
$$

\section{Channel Coding and Image Filtering Techniques}

\section{1. (3, 2) SPC Channel Coding}

In SPC (Single parity check) channel coding, the transmitted binary bits are rearranged into very small code words consisting of merely two consecutive bits. In such $(3,2)$ SPC coding, a single parity bit is added to the message $\mathrm{u}=[\mathrm{u} 0$, $\mathrm{u} 1]$ so that the elements of the resulting code word $\mathrm{x}=[\mathrm{x} 0$, $\mathrm{x} 1, \mathrm{x} 2]$ where, $\mathrm{x} 0=\mathrm{u} 0, \mathrm{x} 1=\mathrm{u} 1$ and $\mathrm{x} 2=\mathrm{u} 0{ }^{\circledR} \mathrm{u} 1$, the symbol ${ }^{\circledR}$ is indicative of the sum over GF (2) [10]

\subsection{D Median Filtering}

2D median filtering is widely used to remove various types of 'pepper' and 'Gaussian' type noises. In this paper 2D Median Filtering scheme is utilized in which a $3 \times 3$ neighborhood windowing mask is used for simply sorting all the pixel values within the window and finding the median value and replacing the original pixel value with the median value [11].

\section{System Description}

The block diagram of the 5G compatible downlink Pattern 
Division Multiple Access (PDMA) scheme implemented wireless communication system model has been presented in Figure 1. In such simulated model, RGB color image will be processed. The RGB color image is different for three users but each is of identical size (260 pixels (width) and 140 pixels (height)). The chosen color image is primarily converted into its respective three Red, Green and Blue components. Its each components is undergone integer to binary converted (not shown in the Figure), channel coded with various Forward Error Correction codes (FEC). The processed binary data are digitally modulated using low order digital modulations, power scaled and phase inserted (in case of signals for user 2 and user 3), serial to parallel converted, OFDM modulated, parallel to serial converted and PDMA encoded prior to transmission from two transmitting antennas of Base station. The signal transmitted from two transmitting antennas are in different resource elements such that the frequency of transmitted carrier is different. In receiving section of each user, own transmitted signal is detected along with other user's signal transmitted in identical resource element considered as interference. The received signal is processed for interference reduction and subsequently sent up to the serial to parallel (S/P) converter, OFDM demodulated, parallel to serially converted, power rescaled and phase removed, digitally demodulated, channel decoded and eventually transmitted color image / binary data are retrieved. Our presently considered simulated system is working in MATLAB environment based on the simulation parameters presented in Table.

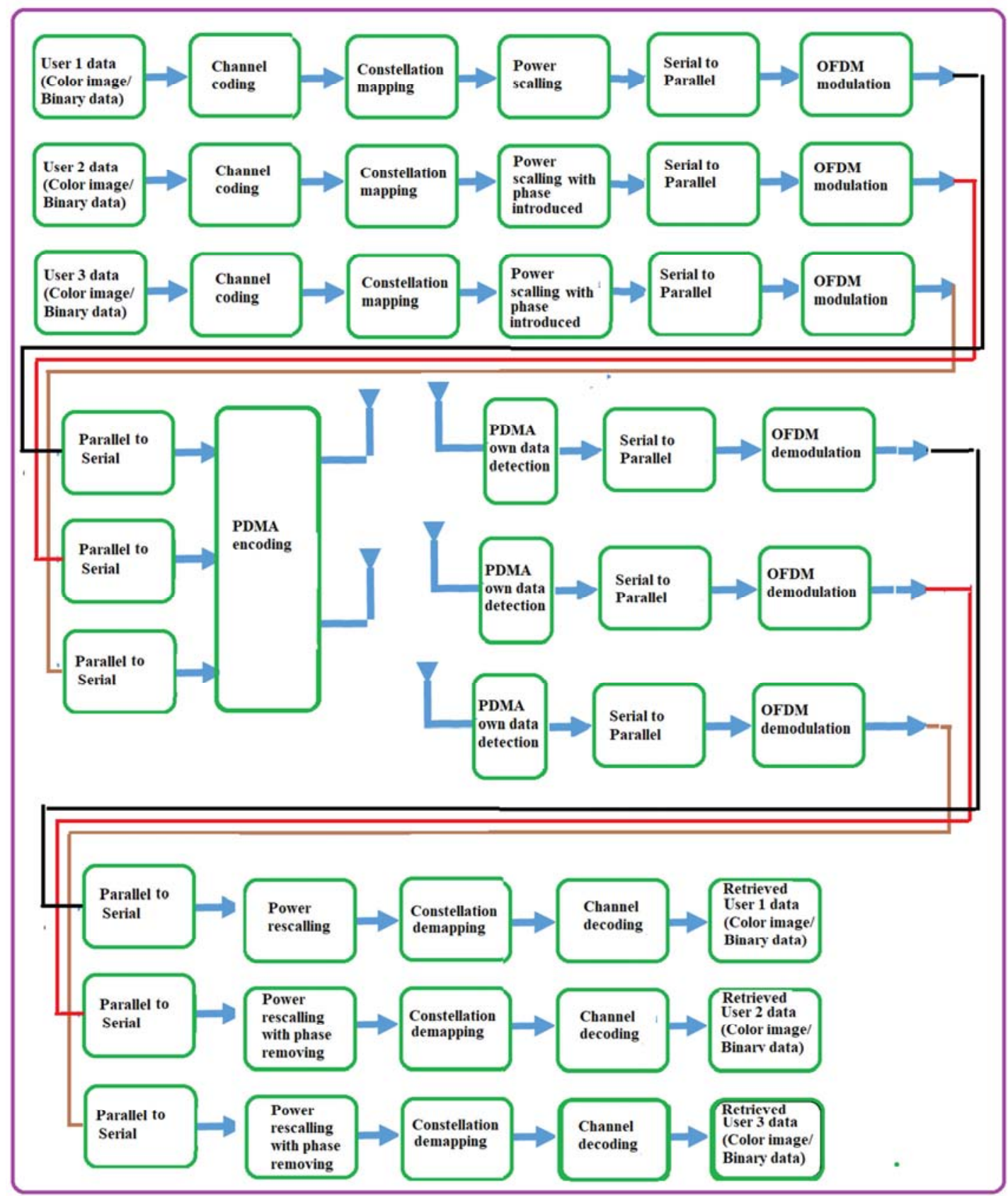

Figure 1. Block diagram of 5 G Compatible Downlink Pattern Division Multiple Access (PDMA) scheme implemented Wireless Communication System. 
Table 1. Summary of the simulated model parameters.

\begin{tabular}{ll}
\hline Parameters & Values \\
\hline Size of Color image for each user & 140 pixels $\times$ 260 pixels \\
PDMA pattern matrix & $\mathrm{G}^{[2,3]}$ PDMA \\
Total transmitted power (Watt) & 40 \\
$\begin{array}{l}\text { Assigned power to user 1, user 2 and user 3 } \\
\text { (of total transmitted power) }\end{array}$ & $50 \%, 30 \%$ and $20 \%$ \\
Phase introduced intransmitted signals of & $\pi$ and $\pi / 4$ \\
user 2 and user 3 & $(3,2)$ SPC \\
Channel encoding & DQPSK, QPSK and QAM \\
Symbol mapping & $2-D$ median \\
Image filter & $0-15$ \\
Signal to noise ratio, SNR $(\mathrm{dB})$ & \\
\hline
\end{tabular}

It is keenly observable from graphical illustrations presented in Figure 2 through Figure 4 that the performance of the downlink $(3,2)$ SPC channel encoded PDMA scheme based $5 \mathrm{G}$ compatible simulated wireless communication system is very much well defined. In all cases, the simulated system shows comparatively better performance in QAM as compared to QPSK and DQPSK digital modulations. On critical observation of simulation curves shown in Figure 2, it is seen that for a typically assumed SNR value of $5 \mathrm{~dB}$, the estimated BER values are $0.1859,0.2462$ and 0.3763 which are indicative of system performance improvements of $1.2201 \mathrm{~dB}$ and $1.8425 \mathrm{~dB}$ in case of QAM as compared to QPSK and DQPSK digital modulations. At 20\% BER value, SNR improvements of $6 \mathrm{~dB}$ and $12 \mathrm{~dB}$ are achieved in case of QAM as compared to QPSK and DQPSK digital modulations.

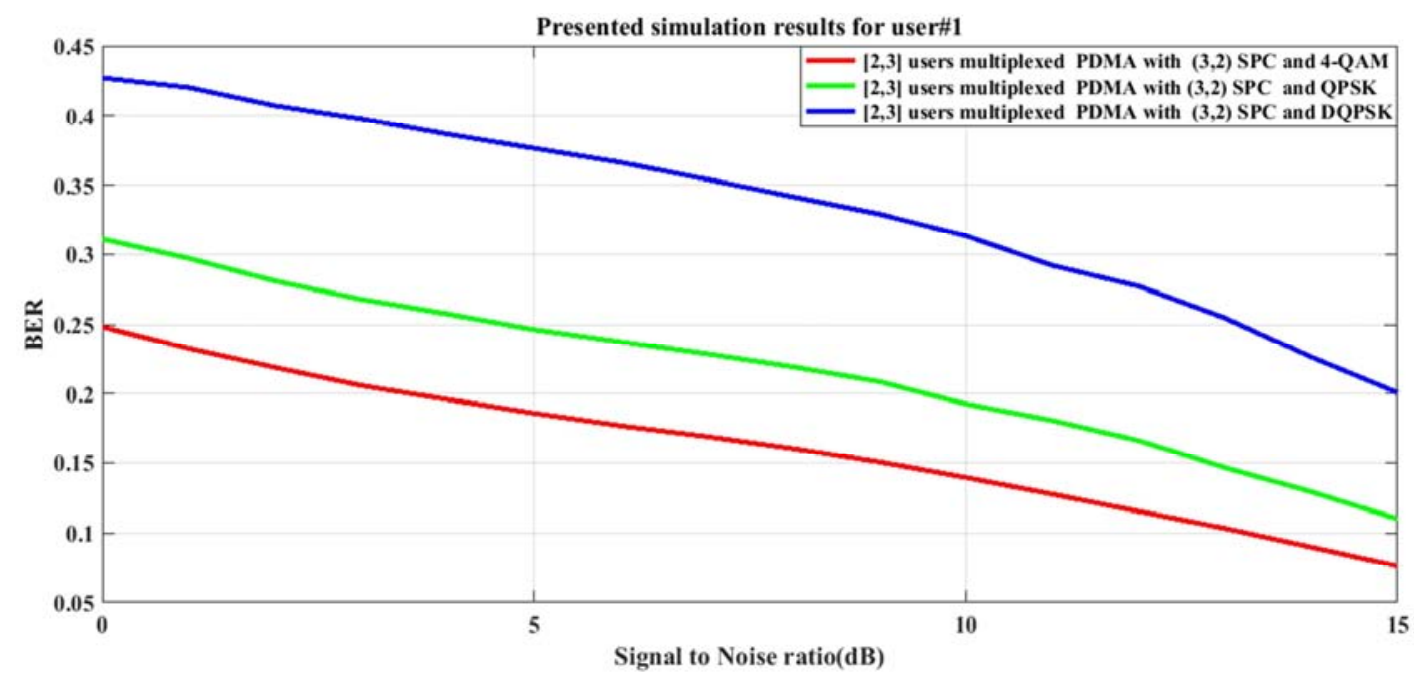

Figure 2. BER performance of (3,2) SPC channel encoded downlink Pattern Division Multiple Access (PDMA) scheme implemented $5 G$ compatible wireless communication system for user \#1 under utilization of various digital modulation schemes.

The BER performance presented in Figure 3 is applicable for user \#2. For a typically assumed SNR value of $5 \mathrm{~dB}$, the estimated BER values are 0.2996, 0.3326 and 0.4513 which ratify system performance improvements of $0.4538 \mathrm{~dB}$ and $1.3254 \mathrm{~dB}$ in case of QAM as compared to QPSK and
DQPSK digital modulation techniques. At 25\% BER value, SNR improvements of $1 \mathrm{~dB}$ and $7 \mathrm{~dB}$ are achieved in case of QAM as compared to QPSK and DQPSK digital modulation techniques.

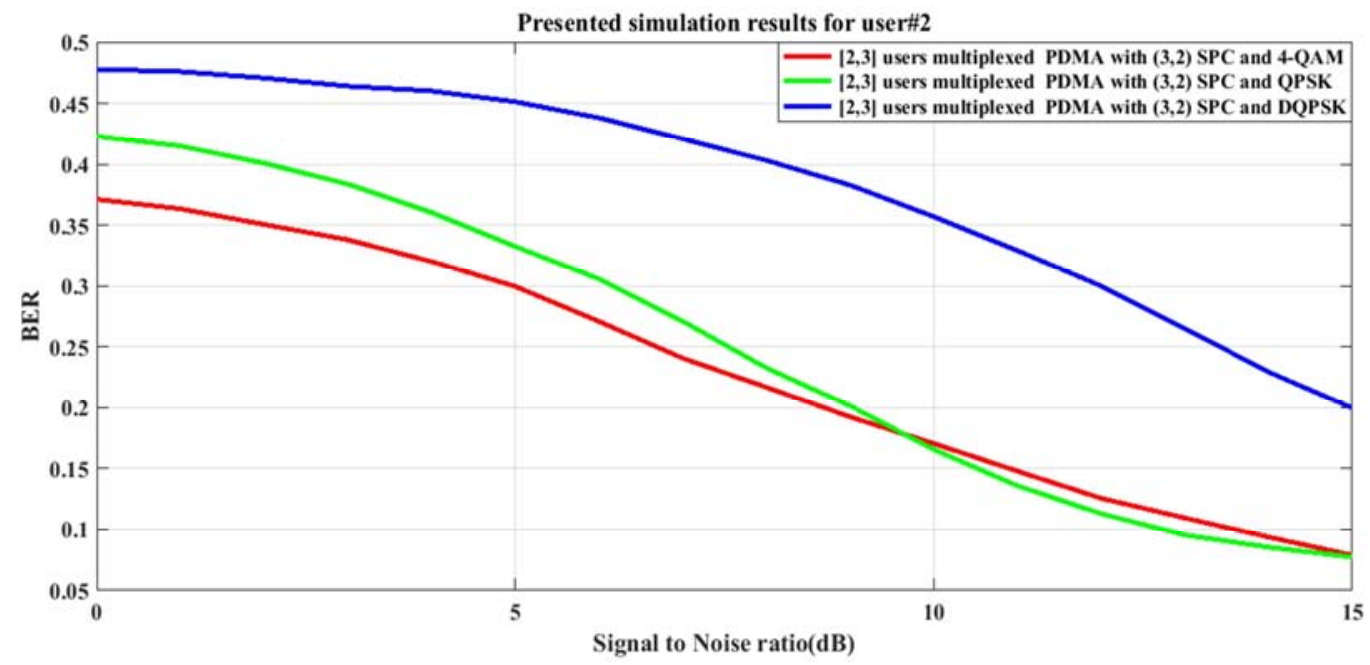

Figure 3. BER performance of (3,2) SPC channel encoded downlink Pattern Division Multiple Access (PDMA) scheme implemented 5G compatible wireless communication system for user $\# 2$ under utilization of various digital modulation schemes. 
On graphical illustration presented in Figure.4, it is observable that the overall system performance is not suitable for user \#3 as power assigned is low as compared to user \#1 and user \#2. For a typically assumed SNR value of $5 \mathrm{~dB}$, the estimated BER values are $0.3204,0.3337$ and 0.4546 which are indicative of system performance improvement of
$0.1766 \mathrm{~dB}$ and $1.3427 \mathrm{~dB}$ in case of QAM as compared to QPSK and DQPSK digital modulation techniques. At 25\% BER value, SNR improvements of $1 \mathrm{~dB}$ and $4 \mathrm{~dB}$ are achieved in case of QPSK in comparison to QAM and DQPSK digital modulation schemes.

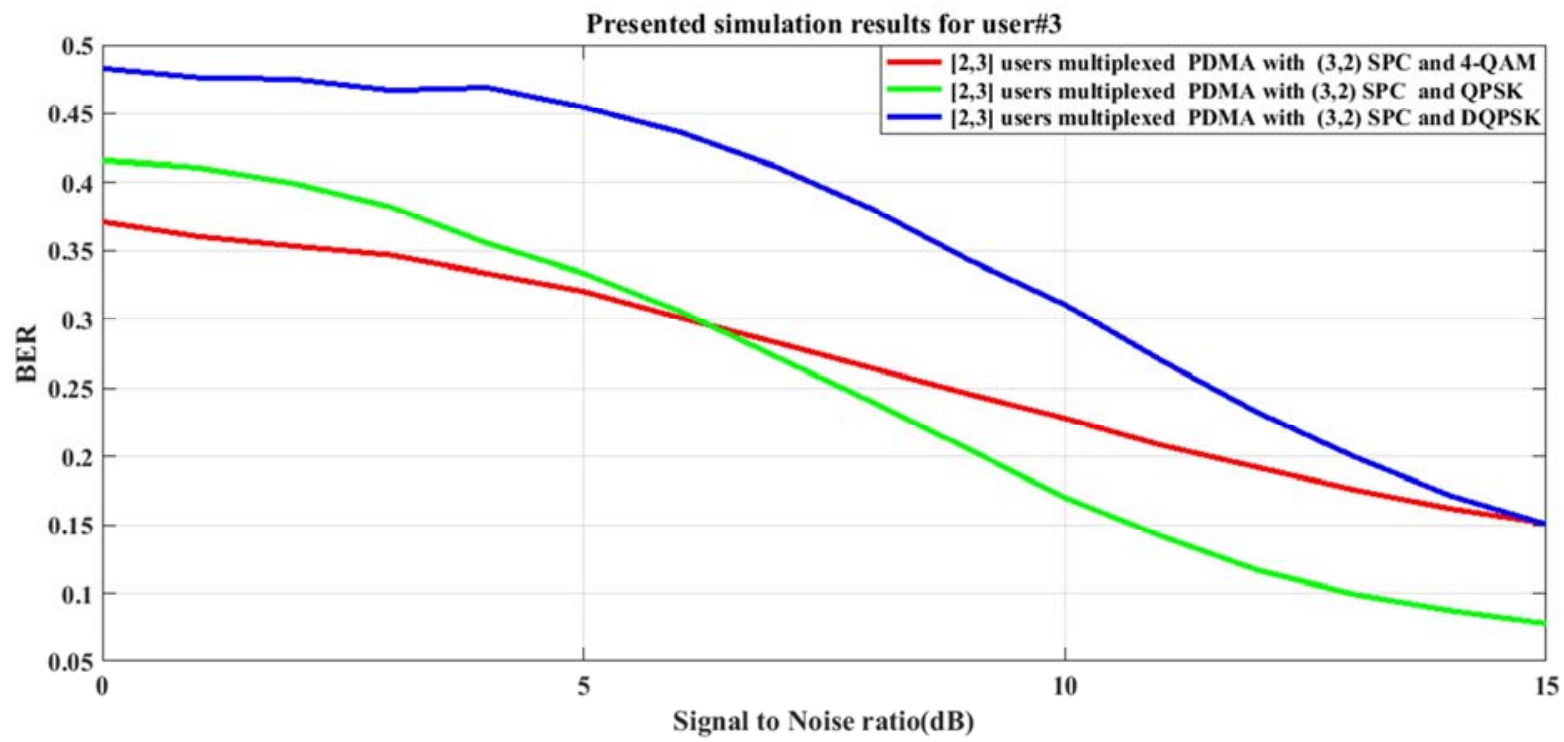

Figure 4. BER performance of (3,2) SPC channel encoded downlink Pattern Division Multiple Access (PDMA) scheme implemented $5 G$ compatible wireless communication system for user \#3 under utilization of various digital modulation schemes.

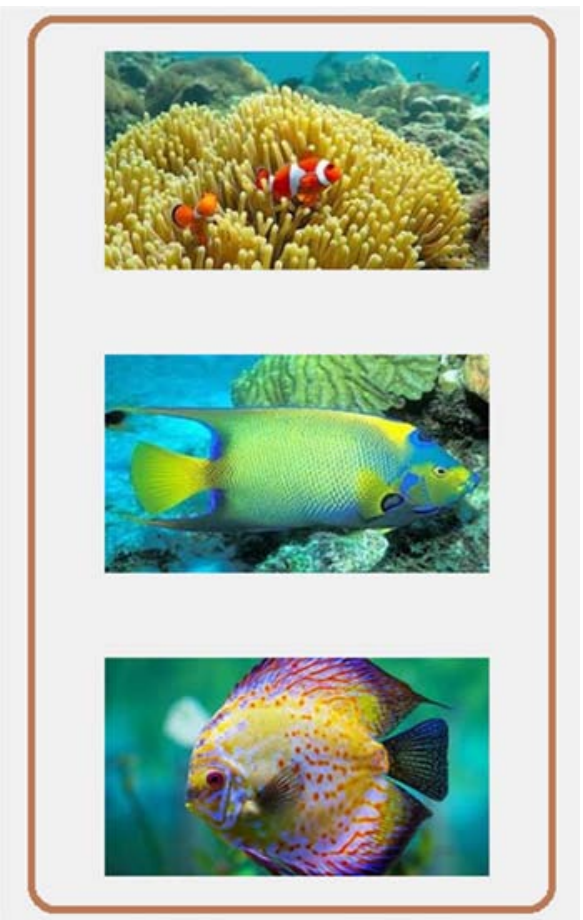

Figure 5. Transmitted color images for three users (Top for user \#1, middle for user \#2 and bottom for user \#3).

Figure 5 shows the three identical sized color images which are considered to have been assigned to the three users. In purpose of showing pixel distributions in the color images, they are converted into gray forms (Figure 6) and corresponding histograms are presented in Figure 7. It is keenly observable that the pixel distributions in the images are not identical to each other. In assigned image for user \#1, the pixel distribution with pixel values in the approximate range: $120-130$ are very much prominent in comparison to others.

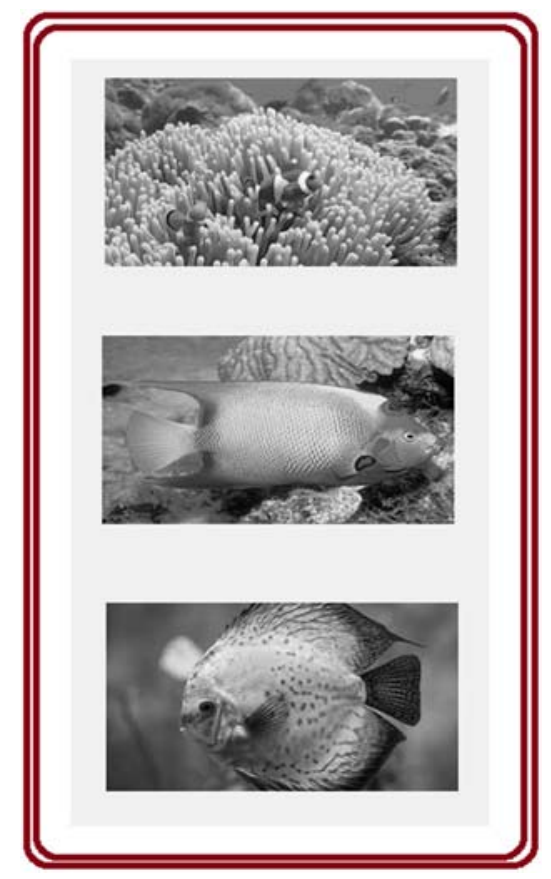

Figure 6. RGB to Gray converted images for three different users. 

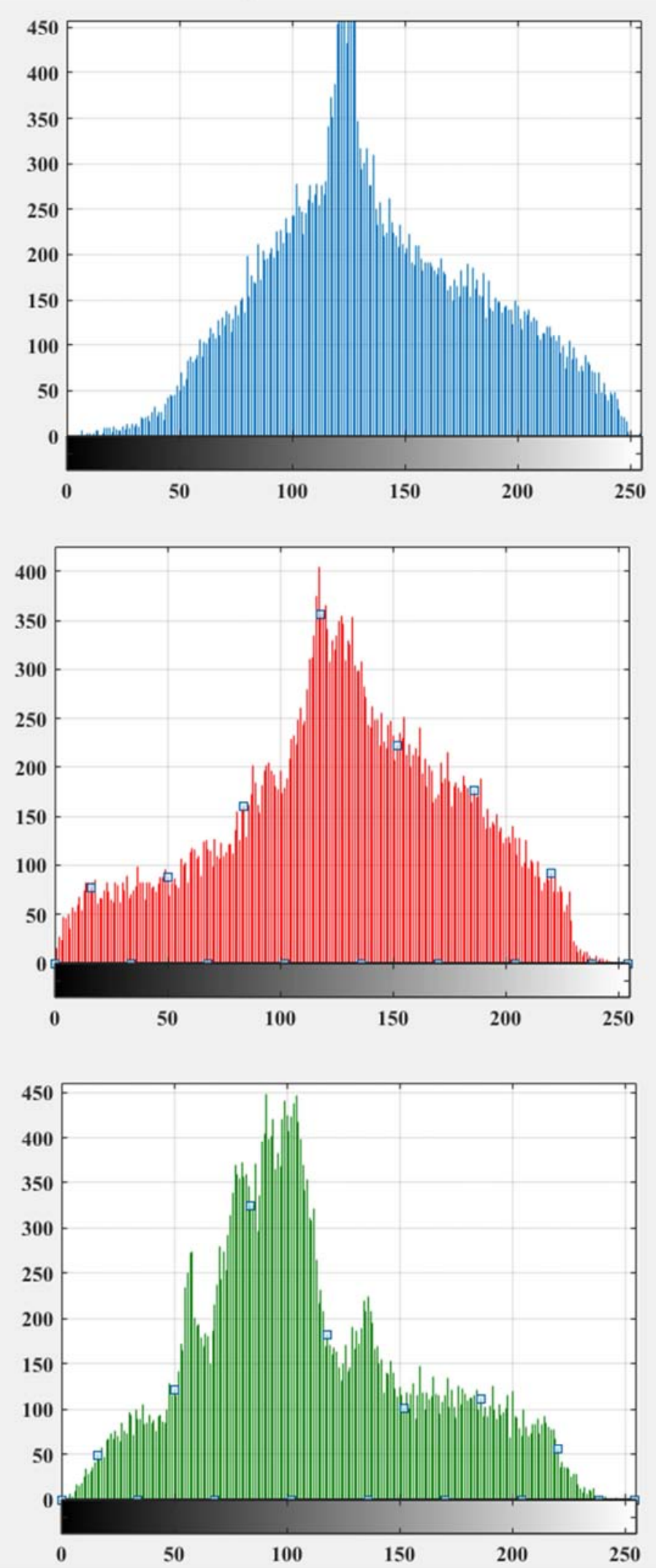

Figure 7. Histograms of RGB to Gray converted images for three different users (Top for user \#1, middle for user \#2 and bottom for user \#3). 
Figure 8 shows received color images for user $\# 1$ at SNR values of $0 \mathrm{~dB}, 5 \mathrm{~dB}, 10 \mathrm{~dB}$ and $15 \mathrm{~dB}$ under scenario of with and without implementation of $2 \mathrm{D}$ image noise reduction technique and utilization of QAM digital modulation in $(3,2)$ SPC channel encoded simulated system. In studying with non using 2D image noise reduction technique, the estimated BER values are found to have values of $0.2566,0.1926$, 0.1442 and 0.0790 in case of $0 \mathrm{~dB}, 5 \mathrm{~dB}, 10 \mathrm{~dB}$ and $15 \mathrm{~dB}$ SNR values. Figure 8 also ratifies that the quality of the retrieved images is improved with application of $2 \mathrm{D}$ image noise reduction technique. Figure 9 shows received color images for user $\# 2$ at SNR values of $0 \mathrm{~dB}, 5 \mathrm{~dB}, 10 \mathrm{~dB}$ and $15 \mathrm{~dB}$ under scenario of with and without implementation of 2D image noise reduction technique and utilization of QAM digital modulation in $(3,2)$ SPC channel encoded simulated system. In studying with non using 2D image noise reduction technique, the estimated BER values are found to have values of $0.0 .3704,0.3030,0.1701$ and 0.0798 in case of $0 \mathrm{~dB}, 5 \mathrm{~dB}$, $10 \mathrm{~dB}$ and $15 \mathrm{~dB}$ SNR values. Figure 9 also ratifies that the quality of the retrieved images is improved with application

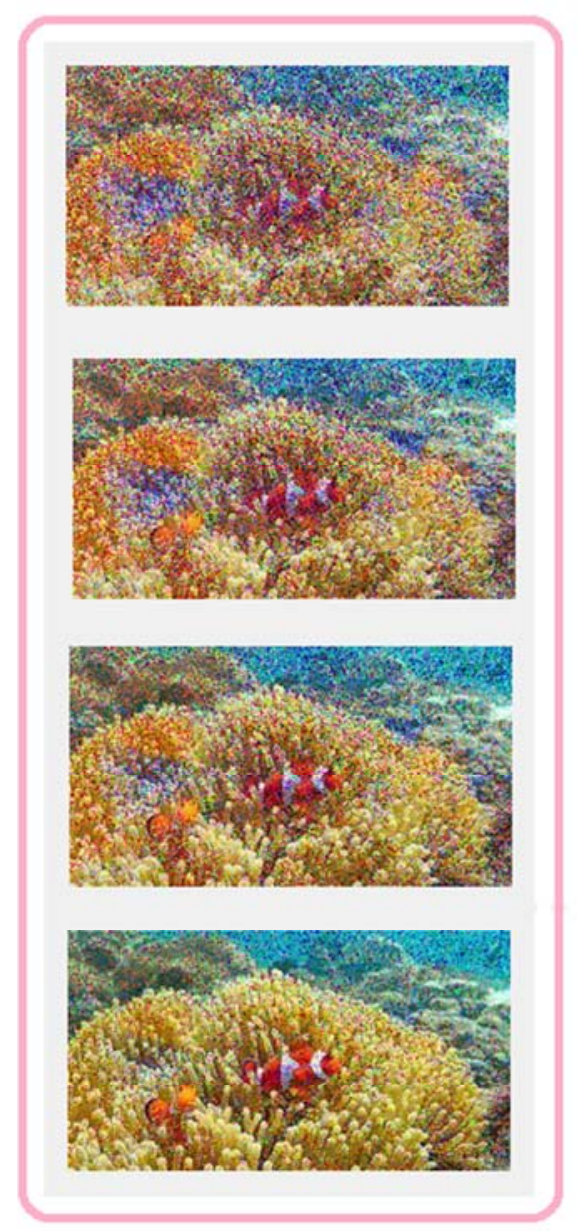

of 2D image noise reduction technique although the $30 \%$ of total power has been assigned to user \#2. Figure 10 shows received color images for user $\# 3$ at SNR values of $0 \mathrm{~dB}, 5$ $\mathrm{dB}, 10 \mathrm{~dB}$ and $15 \mathrm{~dB}$ under scenario of with and without implementation of 2D image noise reduction technique and utilization of QAM digital modulation in $(3,2)$ SPC channel encoded simulated system. In studying with non using 2D image noise reduction technique, the estimated BER values are $0.3726,0.3203,0.2261$ and 0.1513 for SNR values of 0 $\mathrm{dB}, 5 \mathrm{~dB}, 10 \mathrm{~dB}$ and $15 \mathrm{~dB}$. Figure 10 also confirms that the quality of the retrieved images is improved with application of $2 \mathrm{D}$ image noise reduction technique although the $20 \%$ of total power (smallest power) has been assigned to user \#3. Figure 11 through Figure 13 show graphical illustrations for RGB to Gray converted transmitted and retrieved color images with and without filtering at $10 \mathrm{~dB}$ SNR value for different users in $(3,2)$ SPC channel encoded simulated system. It is observable that in each cases presented filtered image has resemblance with the original image.

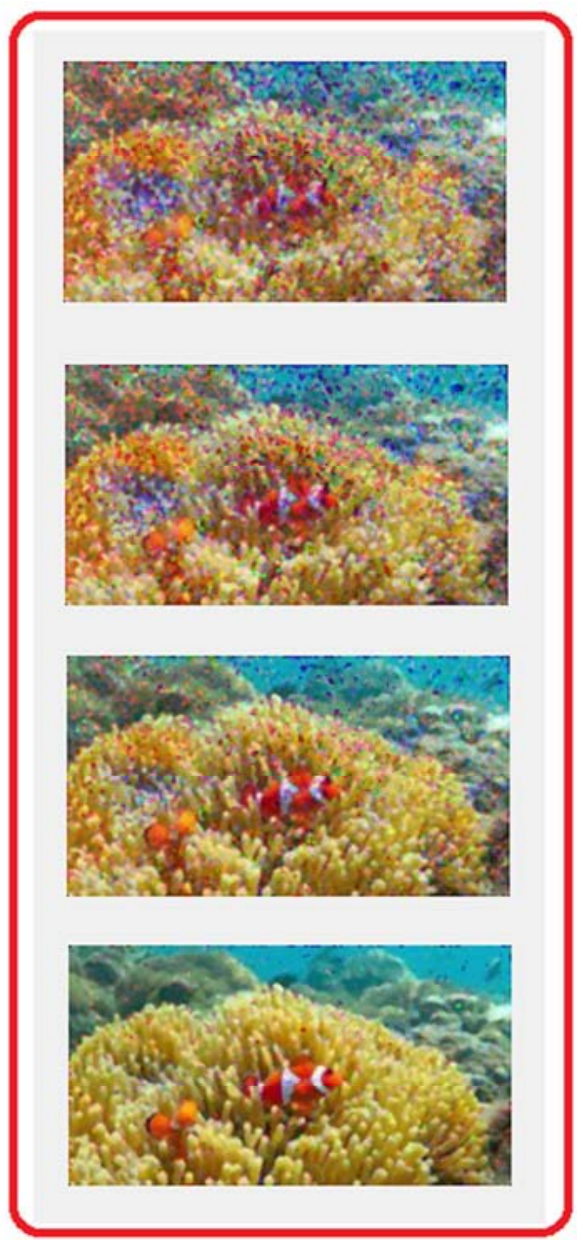

Figure 8. Sequential received color images for user $\# 1$ at SNR values of $0 \mathrm{~dB}, 5 \mathrm{~dB}, 10 \mathrm{~dB}$ and $15 \mathrm{~dB}$ under scenario of with and without implantation of $2 D$ image noise reduction technique (color images presented in Left column and Right column imply the case of without and with implementation of $2 D$ image noise reduction technique). 

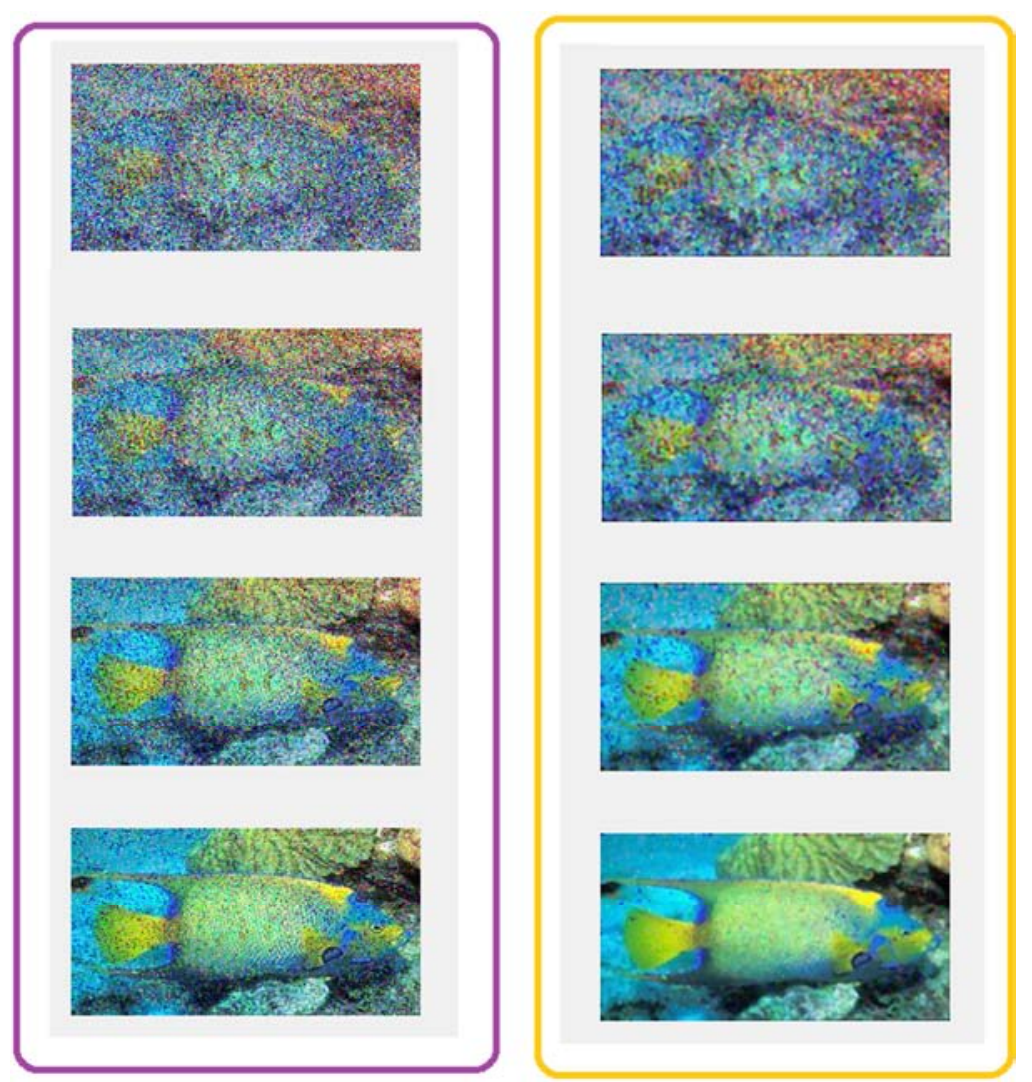

Figure 9. Sequential received color images for user $\# 2$ at $S N R$ values of $0 \mathrm{~dB}, 5 \mathrm{~dB}, 10 \mathrm{~dB}$ and $15 \mathrm{~dB}$ under scenario of with and without implantation of $2 D$ image noise reduction technique (color images presented in Left column and Right column imply the case of without and with implementation of $2 D$ image noise reduction technique).
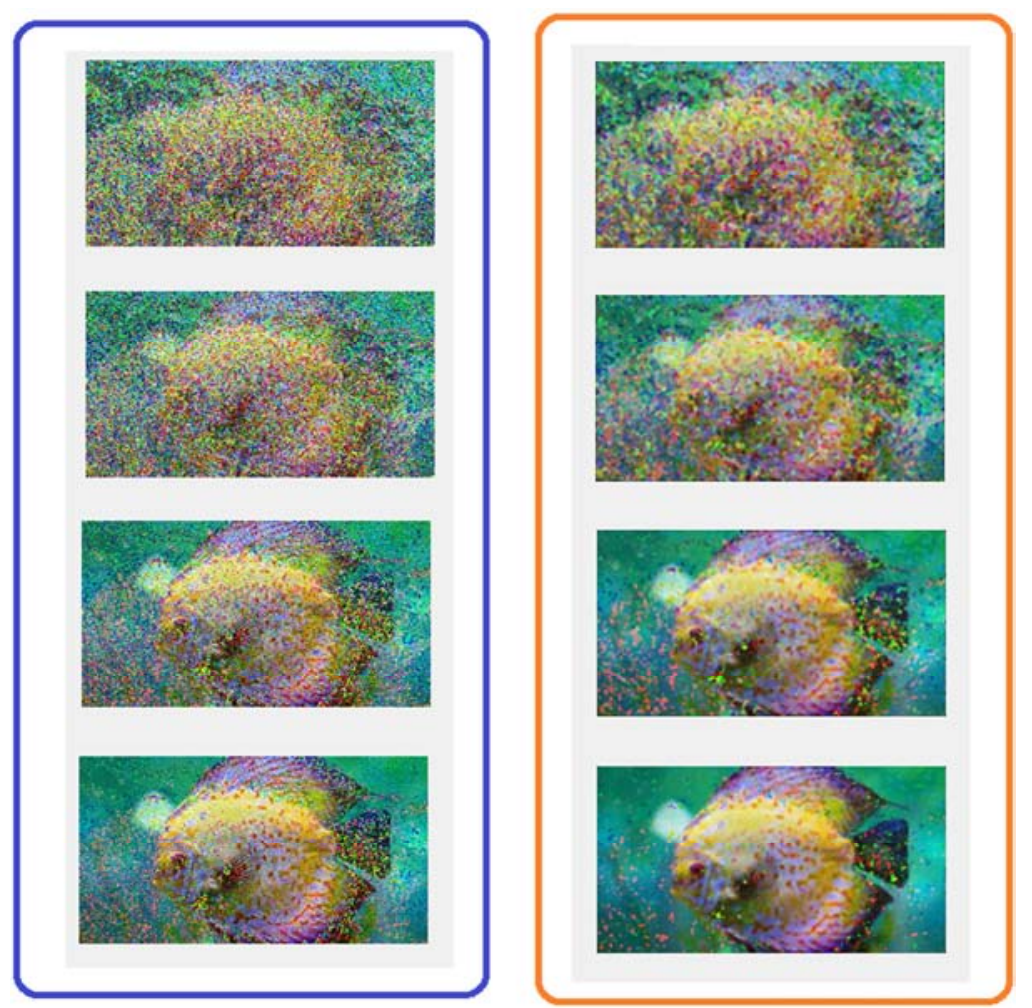

Figure 10. Sequential received color images for user \#3 at SNR values of $0 \mathrm{~dB}, 5 \mathrm{~dB}, 10 \mathrm{~dB}$ and $15 \mathrm{~dB}$ under scenario of with and without implantation of $2 D$ image noise reduction technique (color images presented in Left column and Right column imply the case of without and with implementation of $2 D$ image noise reduction technique). 


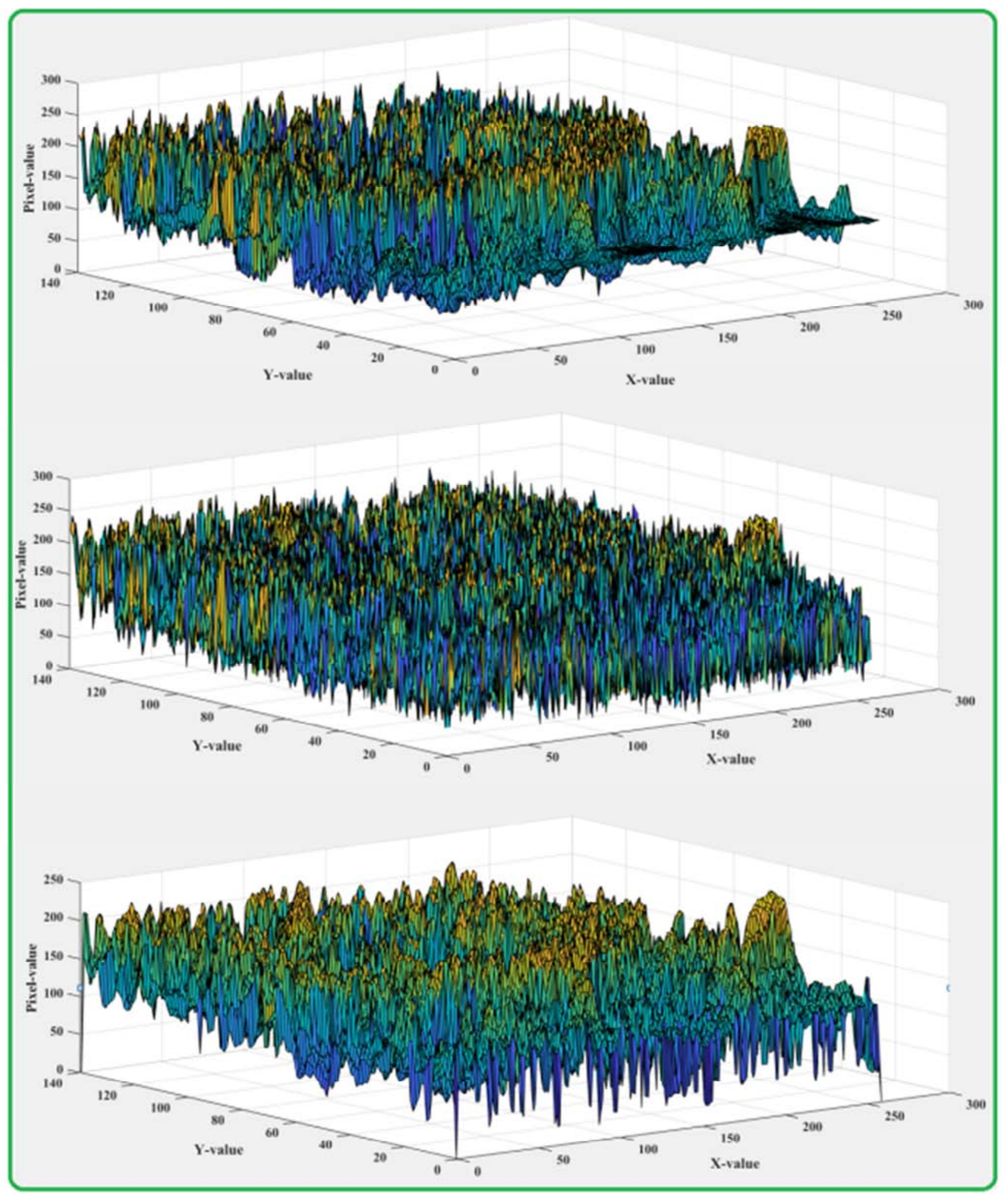

Figure 11. 3-Dimensional Graphical illustrations showing RGB to Gray converted transmitted and retrieved color images with and without filtering at $10 \mathrm{~dB}$ SNR value for user \#1 in (3,2) SPC channel encoded simulated system (Top for original, middle for without filtering and bottom for filtering). 


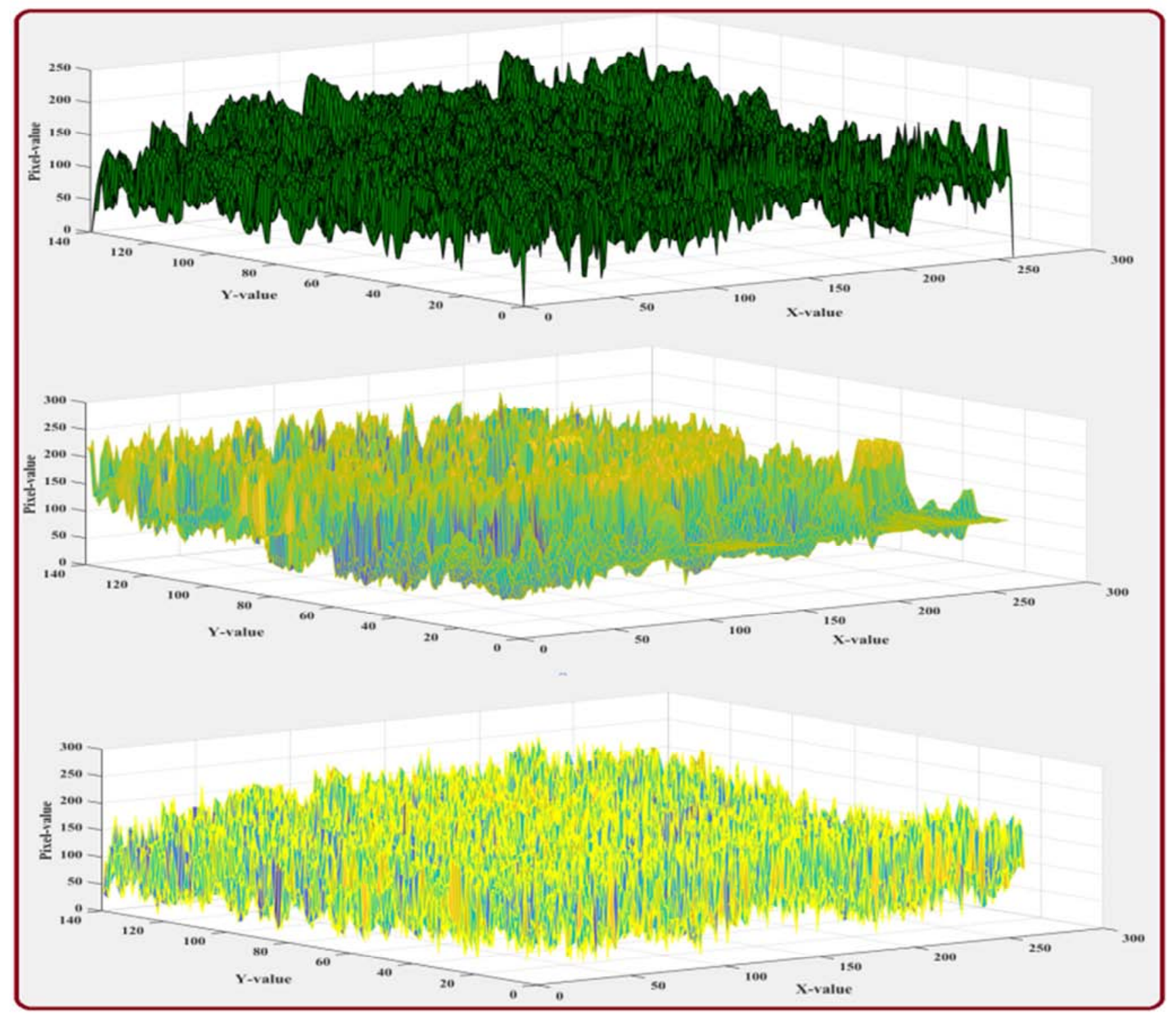

Figure 12. 3-Dimensional Graphical illustrations showing RGB to Gray converted transmitted and retrieved color images with and without filtering at $10 \mathrm{~dB}$ SNR value for user \#2 in (3,2) SPC channel encoded simulated system (Top for original, middle for without filtering and bottom for filtering). 


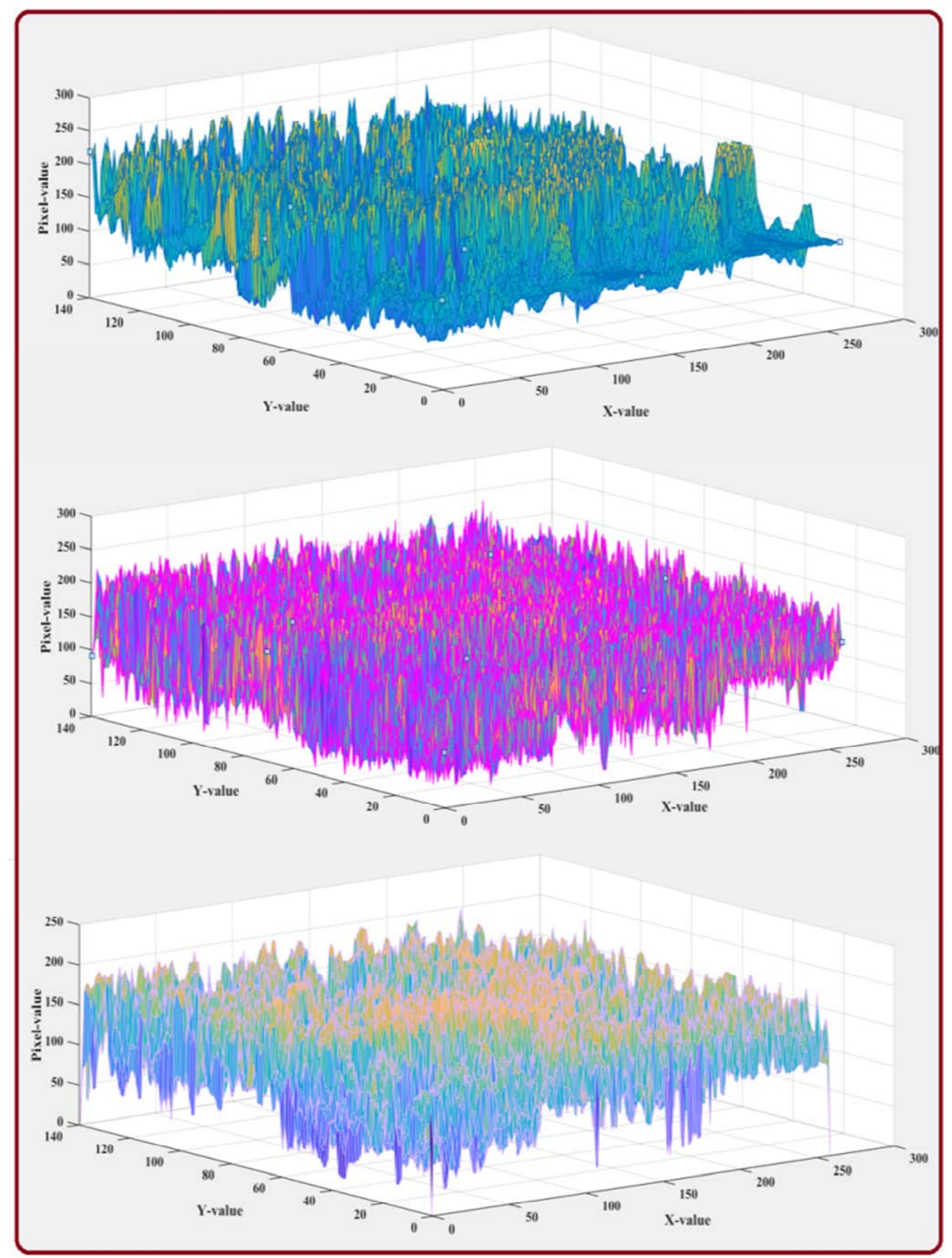

Figure 13. 3-Dimensional Graphical illustrations showing RGB to Gray converted transmitted and retrieved color images with and without filtering at $10 \mathrm{~dB}$ SNR value for user \#3 in $(3,2)$ SPC channel encoded simulated system (Top for original, middle for without filtering and bottom for filtering). 


\section{Conclusions}

In this paper, an comprehensive study has been undertaken on the performance evaluation of 5G Compatible Downlink Pattern Division Multiple Access (PDMA) scheme implemented Wireless Communication System. In such work, an effort has been taken to show the suitability of PDMA scheme in multi-user's data retrieval. To discriminate individual user's signal, phase scaling factors have been introduced and implementation of 2D spatial domain noise reduction technique is found to have produced reasonably watchable color images. Numerical results ratifies the significance of utilizing digital modulation, power assignment and channel coding schemes. In the context of system performance, it can be concluded that the implementation of low order QAM digital modulation technique in $(3,2)$ SPC channel coding scheme provides satisfactory result for such a 5G Compatible Downlink Pattern Division Multiple Access (PDMA) scheme implemented Wireless Communication System.

\section{References}

[1] D. Tse, P. Viswanath, 2005: Fundamentals of Wireless Communication, Cambridge Univ. Press.

[2] IMT-2020(5G) Promotion Group, Whitepapers, [Online]. Available: http://www.imt-2020.org.cn/zh.

[3] $5^{\text {th }}$ Generation Non-orthogonal Waveforms for Asynchronous Signaling,[Online]. Available: http://www.5gnow.eu.
[4] P. Li, L. Liu, K. Wu, W.Leung, 2006: Interleave Division Multiple Access,IEEE Transaction on Wireless Communication., vol. 5, no. 4, pp. 938-947.

[5] H. Jin, K. Peng, J. Song, 2013: Bit Division Multiplexing for Broadcasting, IEEE Trans. on Broadcast., vol. 59, no. 3, pp. 539-547.

[6] Lei Lu; Yan Chen; Wenting Guo; Huilian Yang; Yiqun Wu; Shuangshuang Xing.,2015: Prototype for 5G new air interface technology SCMA and performance evaluation, China Communications. vol.12, pp.38-48.

[7] Zhifeng Yuan; Guanghui Yu; Weimin Li; Yifei Yuan; Xinhui Wang; Jun Xu,.2016: Multi-User Shared Access for Internet of Things, In proceeding of IEEE 83rd Vehicular Technology Conference (VTC Spring).

[8] Linglong Dai; Bichai Wang; Yifei Yuan; Shuangfeng Han; Chih-lin I; Zhaocheng Wang, 2015: Non-orthogonal Multiple Access for 5G: Solutions, Challenges, Opportunities and Future Research Trends, IEEE Communication Magazine,vol. 53, no. 9, pp. 74-81.

[9] Shanzhi Chen, Bin Ren, QiubinGao, Shaoli Kang, Shaohui Sun, and Kai Niu, 2017: Pattern Division Multiple Access (PDMA) -A Novel Non-orthogonal Multiple Access for 5G Radio Networks, IEEE Transactions on Vehicular Technology,vol.66.no.4,pp.3185-3196.

[10] Giorgio M. Vitetta, Desmond P. Taylor, Giulio Colavolpe, Fabrizio Pancaldi and Philippa A. Martin, 2013: Wireless Communications Algorithmic Techniques, John Wiley and Sons Ltd, United Kingdom.

[11] Oge Marques, 2011: Practical Image and Video Processing Using MATLAB, John Wiley and Sons, New Jersey, USA. 